\title{
Whole grain intake in the UK remains low: results from the National Diet and Nutrition Survey rolling programme years 1, 2 and 3
}

Increased whole grain intake has been shown to reduce the risk of many non-communicable diseases such as cardiovascular disease, type 2 diabetes, obesity ${ }^{(1,2)}$ and some cancers ${ }^{(3,4)}$. In response to the evidence, some countries including the US, Canada, Denmark and Australia have dietary guidelines on whole grain intake. However, there is no whole grain specific dietary recommendation in the UK and analysis of dietary survey data from 1986 and 2000 showed that whole grain intake was low and declining in British adults ${ }^{(5)}$. The aim of this study was to describe the whole grain intake in the most current dietary assessment of the UK, The National Diet and Nutrition Survey (NDNS) 2008-11.

The NDNS is a representative dietary survey of households in the UK. Four-day diet diaries were completed by 3073 individuals between 2008 and 2011, along with details of socio-economic status (SES). Daily whole grain intake was calculated for each individual on a dry weight basis. Median whole grain intake was $19.6 \mathrm{~g} / \mathrm{d}$ and $13.4 \mathrm{~g} / \mathrm{d}$ for adults (18+ years) and children/teenagers $(1.5-17$ years), respectively (Fig. 1). Whole grain intake increased with age but was lowest in teenagers (13-17 years) and younger adults up to age 34 years, for whom intakes were lower than those in all other age groups. There were $18 \%$ and $15 \%$ non-consumers of any wholegrain foods, for adults and children/teenagers, respectively. After adjustment for energy intake, adult females had higher whole grain intake than males. Those from lower SES groups had significantly lower whole grain intake than those more advantaged classifications.

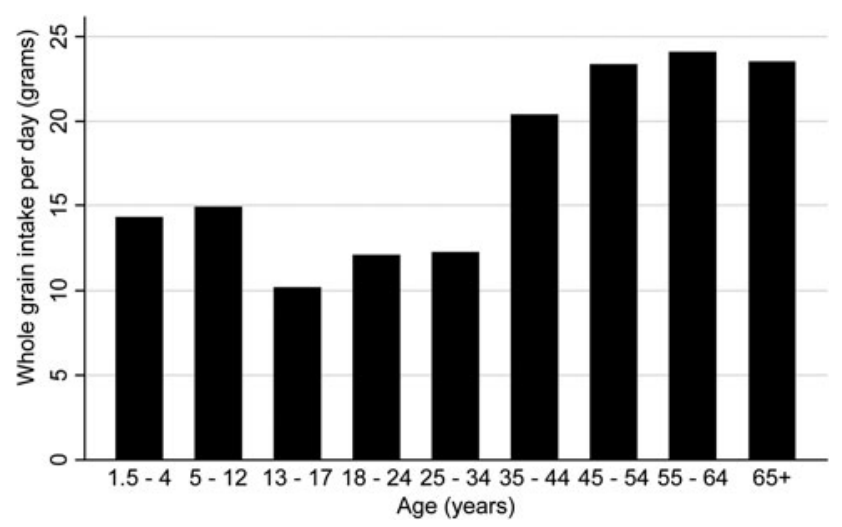

Fig. 1. Median whole grain intake by age

Whole grain intake in this representative UK population was low. Intake was slightly higher than reported in the 2000/01 NDNS $\left(14 \mathrm{~g} / \mathrm{d}\right.$ for adults over $\left.19.6 \mathrm{~g} / \mathrm{d}^{(5)}\right)$. Teenagers and younger adults may need targeting to help increase whole grain consumption. Reducing the cost and increasing the availability of wholegrain foods may help to increase whole grain intake in the UK and other countries without whole grain dietary recommendations. Further investigation into the associated health benefits with whole grain intake in this population is needed.

1. Cho SS, Qi L, Fahey GC Jr. et al. (2013) Am J Clin Nutr 98, 594-619.

2. Ye EQ, Chacko SA, Chou EL et al. (2012) J Nutr 142, 1304-13.

3. Aune D, Chan DS, Lau R et al. (2011) BMJ 343:d6617.

4. Kyrø C, Skeie G, Loft S et al. (2013) Cancer Causes Control 24, 1363-1374.

5. Thane CW, Jones AR, Stephen AM et al. (2007) Br J Nutr 97, 987-992. 